\title{
Storage or retrieval deficit: The yin and yang of amnesia
}

\author{
Oliver Hardt, ${ }^{1,3}$ Szu-Han Wang, ${ }^{1,2,3}$ and Karim Nader ${ }^{1,4}$ \\ ${ }^{1}$ Department of Psychology, McGill University, Montreal, Quebec H3A 1B1, Canada; ${ }^{2}$ Centre for Cognitive and Neural Sciences, \\ Edinburgh University, Edinburgh EH89JZ, United Kingdom
}

\begin{abstract}
To this day, it remains unresolved whether experimental amnesia reflects failed memory storage or the inability to retrieve otherwise intact memory. Methodological as well as conceptual reasons prevented deciding between these two alternatives: The absence of recovery from amnesia is typically taken as supporting storage impairment interpretations; however, this absence of recovery does not positively demonstrate nonexistence of memory, allowing for alternative interpretations of amnesia as impairment of memory retrieval. To address this shortcoming, we present a novel approach to study the nature of amnesia that makes positive, i.e., falsifiable, predictions for the absence of memory. Applying this paradigm, we demonstrate here that infusing anisomycin into the dorsal hippocampus induces amnesia by impairing memory storage, not retrieval.
\end{abstract}

Amnesia-inducing treatments impair performance on subsequent retention tests when administered shortly after learning but not when applied after a delay (cf. Davis and Squire 1984). Although studied for several decades now, it has never been conclusively determined whether this deficit represents disrupted memory storage or a failure in retrieving otherwise intact memory (see debate in Learning \& Memory, Volume 13, No. 5 [http:// learnmem.cshlp.org/content/13/5.toc]). Given that understanding amnesia has widespread implications ranging from basic theory formation to clinical application and jurisdiction, a resolution of this question seems timely and desirable.

Two orthogonal interpretations exist for memory recovery after amnesia induction, and the currently available evidence does not allow deciding between them (Nader and Wang 2006; Squire 2006). If, as the storage impairment view suggests, amnesia reflects actual memory loss, then no recovery should ever be seen. But if performance never recovers, it will not falsify retrieval impairment explanations-one cannot rule out that the retrieval blockade is too strong or that the proper retrieval cues that might recover memory have not been used. On the other hand, successful recovery does not unequivocally support the retrieval-failure position: Since experimental manipulations never induce complete amnesia, reminders may lead to recovery because they promote new learning adding onto a residual memory trace (Gold and King 1974). It should be noted, however, that the new learning hypothesis cannot readily explain some instances of recovery, when, for example, memory recovers after re-exposure to the training context in the absence of the conditioned stimulus (Sara 1973), after administration of an extinction trial (Gordon and Mowrer 1980), or after exposure to a novel spatial location such that the old position could not be reacquired (de Hoz et al. 2004). In summary, both positions can explain most outcomes of experiments that use memory recovery as a criterion for the presence or absence of memory after amnesia induction (Table 1).

To resolve this stalemate, a paradigm is needed that (1) allows the storage impairment view to positively demonstrate the

\footnotetext{
${ }^{3}$ These authors contributed equally to this work.

${ }^{4}$ Corresponding author.

E-mail karim.nader@mcgill.ca; fax (514) 398-4896.

Article is online at http://www.learnmem.org/cgi/doi/10.1101//m.1267409.
}

absence of memory, which is not equal to the absence of recovery, and (2) equips retrieval and storage views of amnesia with opposing predictions, making empirical disambiguation possible. We here describe a method that meets both requirements (Table 2). Our paradigm exploits the fact that $N$-methyl-D-aspartate receptors (NMDAr) are required in the dorsal hippocampus (dHPC) only when contextual fear conditioning is learned for the first, but not the second time (Sanders and Fanselow 2003; see also Bannerman et al. 1995). The requirement for NMDAr in dHPC during the second learning can thus be used to assess whether memory for a previous experience exists. If inducing amnesia for the first contextual fear conditioning impairs storage, then corresponding memory should not be available at the time of the second training. Consequently, NMDAr antagonists, such as 2-amino-5-phosphonopentanoic acid (AP5), should block acquisition of the second contextual fear, as this then represents the first instance of such learning because no memory for the previous conditioning is present. On the other hand, retrieval accounts of amnesia assume that memory for the first learning is stored but cannot be retrieved; hence, memory for the first contextual fear should exist when the second contextual fear is acquired. Therefore, learning of the second contextual fear should not be blocked by dHPC infusions of AP5.

Using this paradigm, we tested whether infusing the proteinsynthesis inhibitor anisomycin (ANI) into dHPC after contextual fear conditioning disrupts memory storage or retrieval.

\section{Results}

Intra-dHPC AP5 blocks only the first, but not the second, contextual fear conditioning

Using the protocol described by Sanders and Fanselow (2003), which leads to little generalization between the contexts (see Methods), animals were bilaterally infused with either AP5 or its vehicle (VEH) into the dHPC before the first contextual fear conditioning (Fig. 1A). A retention test administered the following day suggested that blocking NMDAr prevented context fear learning, as AP5-treated animals froze significantly less than the vehicle-injected controls, $F_{(1,20)}=33.12, P<0.0001$ (Fig. 1B).

Four days later, animals were fear conditioned to a different context. Before training, half of the animals in each group received 
Table 1. The specific arguments invoked in the recovery from amnesia paradigm to explain the nature of amnesia that brought the debate to a standstill

\begin{tabular}{ll}
$\begin{array}{l}\text { Recovery from } \\
\text { amnesia observed? }\end{array}$ & \multicolumn{1}{c}{ Storage impairment view } \\
\hline Yes & $\begin{array}{c}\text { Consistent, as recovery reflects new learning } \\
\text { added onto residual memory. } \\
\text { Consistent with storage impairment. }\end{array}$ \\
No & $\begin{array}{l}\text { Generates only "negative" predictions } \\
\text { (i.e., no recovery from amnesia) and cannot } \\
\text { prove that memory does not exist. }\end{array}$ \\
$\begin{array}{l}\text { Theoretical } \\
\text { shortcomings }\end{array}$
\end{tabular}

${ }^{a}$ The storage impairment view can explain recovery from amnesia because amnesia is never complete-some residual performance always remains, suggesting that some part of the memory was still stored. Recovery thus is attributed to new learning or modulation of the residual trace (Gold and King 1974; Nader and Wang 2006). Thus, both views can explain any outcome of the recovery from amnesia paradigm, the dominant method used to study the nature of amnesia.

${ }^{\mathrm{b}}$ In an attempt to go beyond repeated demonstrations of negative findings to seek support for the storage impairment view of amnesia, some took reductionist approaches (Bailey et al. 1996; Squire 2006). These entail identifying molecular, morphological, or cellular correlates observable after learning, assuming that they reflect memory storage processes. Amnesia-inducing treatments that block the induction of these changes are interpreted as impairing storage. However, some behavioral models of memory posit that memory storage occurs within seconds after learning and that the postlearning correlates of plasticity reflect the establishment of retrieval mechanisms alone (e.g., Lewis 1979; Miller and Marlin 1984): The absence of the correlates thus reflects impaired retrieval, not disrupted storage. Some support for this view comes from recent demonstrations (in Aplysia) that even after memory is lost, physiological changes remain that can facilitate subsequent new learning (Philips et al. 2006).

AP5, the other half vehicle in the dHPC (these groups will be referred to as VEH-VEH, VEH-AP5, AP5-VEH, and AP5-AP5; the abbreviations refer to the drug received before the first and second conditioning, respectively). To ensure that differences in memory for the first context fear cannot account for any second-learning effects, animals were pseudorandomly assigned to each group, with the constraint that freezing to the first context was similar between them (i.e., VEH-VEH and VEH-AP5 showed similar retention of the first learning, as did AP5-VEH and AP5-AP5, $t_{(9)}=$ $0.82, P=0.43$, and $t_{(9)}=-0.12, P=0.91$, respectively, Fig. $\left.1 \mathrm{C}\right)$.

Only animals that received AP5 before each training session had impaired memory for the second training (Fig. 1A, right graph: Group AP5-AP5 performed worse than all other groups; post hoc tests revealed $P<0.03$ for all comparisons analyzing the significant interaction, $\left.F_{(1,18)}=5.36, P<0.04\right)$. Performance of the other groups was intact and at the same level, $P>0.55$. Thus, group VEH-AP5 readily learned to fear the second context although animals received AP5 infusions before the second training, a treatment that impaired learning when applied before the first training.

These findings replicate that AP5 blocks learning only when a task is acquired for the first time (Bannerman et al. 1995; Sanders and Fanselow 2003). The effect cannot be attributed to unspecific effects of AP5 on, for example, attention, motivation, or perception. If this were the case, then animals that received AP5 during the second session (VEH-AP5) should not have acquired contextual fear, which they did.

To conclude, this paradigm provides sensitivity of learning to intra-dHPC AP5 as a tool for testing the presence or absence of existing memory of a previous experience: Only the second instance of contextual fear conditioning is unaffected by the very drug infusions that impair the first learning.

\section{Intra-dHPC AP5 blocks the second contextual fear learning after ANI infusions impaired memory for the first one}

Immediately after fear conditioning to the first context, animals received dHPC infusions of ANI or its vehicle (Fig. 2A). The retention test the following day revealed the typical memory impairment found after ANI infusion: Rats in the ANI group froze significantly less to the conditioning context than the vehicleinfused controls, $F_{(1,20)}=14.19, P<0.001$ (Fig. 2B). The point of interest now is whether this memory impairment is due to blocking storage or retrieval processes. If ANI infusion disrupted memory storage, then memory for the first conditioning should be absent in the amnesic animals. Consequently, these animals should "treat" the second learning as if it was the first time they were contextually fear conditioned. Hence, intra-dHPC AP5

Table 2. A paradigm that makes positive predictions for the absence of memory

\begin{tabular}{|c|c|c|c|}
\hline & \multirow[b]{2}{*}{ Control animals } & \multicolumn{2}{|c|}{ For animals that are amnesic for the first learning } \\
\hline & & Storage impairment view & Retrieval impairment view \\
\hline $\begin{array}{l}\text { Specific prediction } \\
\text { for the second learning }\end{array}$ & $\begin{array}{l}\text { Unaffected by AP5 } \\
\text { infusions into dHPC }\end{array}$ & $\begin{array}{l}\text { If amnesia }{ }^{\text {a }} \text { represents impaired storage } \\
\text { of the first memory, then the second } \\
\text { learning should be treated as if it was } \\
\text { the first one. Hence, it should be blocked } \\
\text { by dHPC-AP5 - a positive prediction. }{ }^{\mathrm{b}}\end{array}$ & $\begin{array}{l}\text { If the first memory exists but cannot be } \\
\text { retrieved, then the second learning should } \\
\text { still be treated as the first one. Hence, it should } \\
\text { be unaffected by dHPC-AP5. }\end{array}$ \\
\hline
\end{tabular}

a"Amnesia" does not refer to a comprehensive impairment affecting all aspects of the memory distributed across the brain. The question is whether storage or retrieval is impaired in the targeted memory system. Thus, amnesia, as used in the studies reported here, refers exclusively to dHPC-mediated representations and processes involved in contextual fear conditioning.

bWile the mechanisms mediating the second learning are unknown, the logic of our experiments does not rest on the specifics of these mechanisms. Rather, our predictions address, independent of the nature of the second learning, whether amnesia renders the second learning sensitive to those manipulations that have been shown to only affect the first learning. 
A

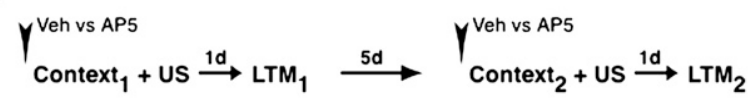

B

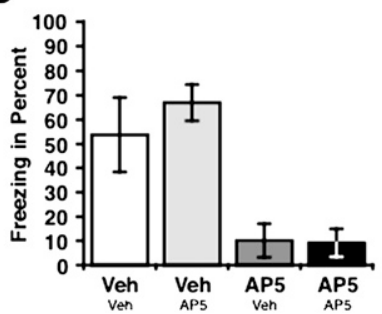

C

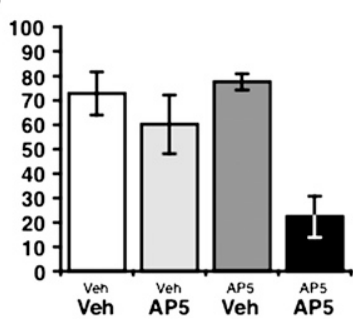

Figure 1. Experiment 1. Intra-dHPC AP5 blocks only the first, but not the second, contextual fear conditioning. $(A)$ Experimental protocol. Upside-down arrows indicate drug and time of infusion into the dHPC. $(B)$ Long-term memory for the first contextual fear conditioning (LTM1 in percent freezing, error bars indicate SEM). AP5 impairs the first learning of contextual fear (LTM1 for groups AP5-VEH and AP5-AP5). (C) AP5 does not block acquisition of the second contextual fear in animals with intact memory for the first conditioning (compare LTM2 for groups VEH-AP5 and AP5-AP5).

should block learning of the second contextual fear in these animals. If, on the other hand, ANI disrupted retrieval, such that memory for the first learning exists at the time of the second conditioning, intra-dHPC AP5 should not block the second learning (see Table 2).

Four days after the first retention test, animals were fear conditioned to the second context, exactly as in Experiment 1. Half of the animals of each group were either infused with AP5 or VEH before the second learning (these groups will be referred to as VEH-VEH, VEH-AP5, ANI-VEH, and ANI-AP5). Animals were pseudorandomly assigned to each group, such that freezing to the first context was similar between them (i.e., VEH-VEH and VEH-AP5 showed similar retention of the first learning, $t_{(8)}=0.33$, $P=0.75$, as did ANI-VEH and ANI-AP5, $t_{(10)}=-0.46, P=0.65$, Fig. 2B).

Memory retention was assessed $1 \mathrm{~d}$ after the second conditioning session (Fig. 2C). Animals with amnesia for the first contextual fear did not acquire fear to the second context if they received AP5 infusions (i.e., ANI-AP5 performed worse than all the other groups, $P<0.04$ for all comparisons analyzing the significant interaction, $\left.F_{(1,18)}=4.68, P<0.05\right)$. Together with the finding that the amnesic animals that received vehicle prior to the second learning (ANI-VEH) performed as well as those that had intact fear memory for the first context (i.e., VEH-VEH and VEH-AP5 were not significantly different from ANI-VEH, $P>0.9$ for all comparisons), this result indicates that learning of the second contextual fear was blocked by dHPC AP5 infusions in animals with ANIinduced amnesia for the first contextual fear learning. At the end of the experiment, ANI-AP5 rats were retrained in a third context and showed comparable learning to the VEH-VEH group, $P=0.64$ (data not shown). Hence, it is unlikely the infusion of ANI and AP5 caused comprehensive hippocampal damage.

In summary, concerning this task, infusing ANI into the dHPC after contextual fear conditioning induces a memory deficit that makes subsequent contextual fear learning sensitive to AP5. As we have shown in Experiment 1, only the first, but not the second, learning of contextual fear is blocked by AP5 infusion in the dHPC. Amnesic animals thus treated the second conditioning as if they acquired this task for the first time. Therefore, inducing amnesia for contextual fear by infusing ANI in the dHPC appears to cause actual memory loss.

Intra-dHPC AP5 does not block the second contextual fear learning after extinguishing the first one

Post hoc, one could argue that AP5 impaired the second learning in Experiment 2 because the memory or conditioned response for the first conditioning could not be retrieved, although it was present. If this interpretation holds, then inhibiting the expression of the first conditioned response should affect the second learning in the same way, i.e., it should be blocked by AP5.

To test this prediction, we used extinction to create a state in which existing memory or the response for the first conditioning is no longer expressed. Delayed extinction is thought to result in memory, or response inhibition, not actual memory loss (e.g., Rescorla 2000; Bouton 2004). Although it could be argued that extinction represents a rather poor method to study the molecular requirements of the second learning following certain forms of amnesia induction, extinction is used here as a tool to induce retrieval inhibition without affecting the integrity of the actual memory. This allows investigating whether the mechanisms of the second learning will change when memory is not retrieved or expressed although it still exists.

According to our interpretation of the results of Experiment 2 , the effects of extinguishing the first conditioning on learning the second one should be opposite to the effects that were obtained after inducing amnesia by ANI- or AP5-infusions (Table 2): Since memory for the first learning should still be available after delayed extinction, the second learning of contextual fear should not be blocked by AP5. If, on the other hand, the alternative explanation outlined above is correct, then AP5 should block the second learning, just as it did in Experiment 2 .

As in the previous experiments, animals were fear conditioned to the first context (Fig. 3A). All animals received vehicle injections before training, to keep the procedure consistent with the previous experiments. As before, animals were then pseudorandomly assigned to two groups, such that both showed comparable freezing, $t_{(6)}=0.8, P=0.44$.

Fear to the first context was then extinguished (Fig. 3B) by exposing the animals to the conditioned stimulus (i.e., the context) in the absence of the unconditioned stimulus (i.e., the foot shock). The retention test $1 \mathrm{~d}$ after training presented thus the first extinction trial. During the following $4 \mathrm{~d}$, animals were daily exposed to an extinction session. The fear response to the first

A

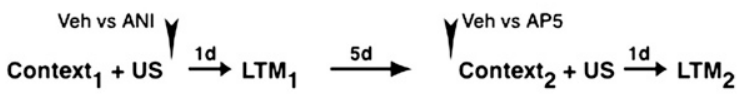

B

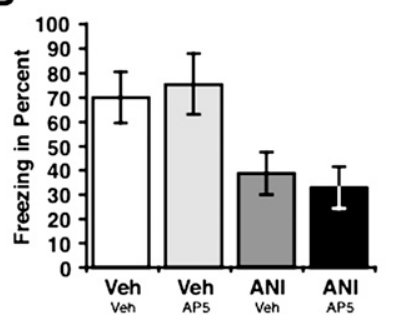

C

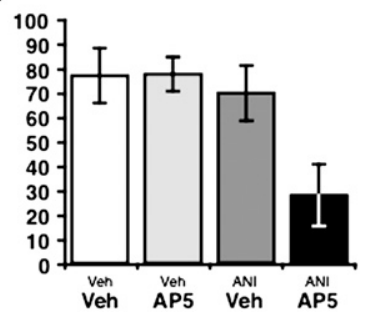

Figure 2. Experiment 2. Intra-dHPC AP5 blocks the second contextual fear learning after $\mathrm{ANI}$ infusions impaired memory for the first one. $(A)$ Experimental protocol. (B) ANI infusions after the first contextual fear conditioning impair long-term memory (LTM1 for groups ANI-VEH and ANI-AP5). (C) Animals amnesic for the first contextual fear conditioning following ANI infusions did not acquire the second contextual fear when AP5 was infused into dHPC prior to the second conditioning (compare LTM2 for groups VEH-AP5 and ANI-AP5). 
A

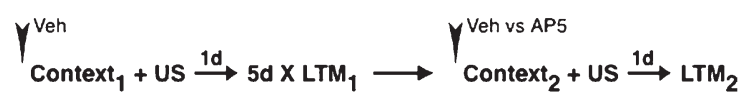

B

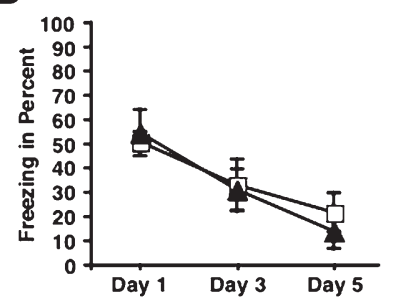

C

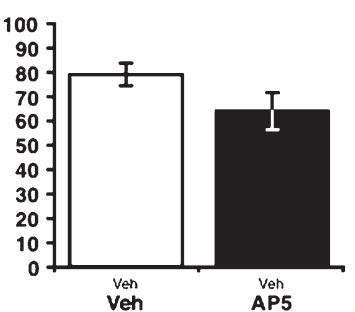

Figure 3. Experiment 3. Intra-dHPC AP5 does not block the second contextual fear learning after extinguishing the first one. $(A)$ Experimental protocol. (B) A daily exposure to the training context in the absence of foot shock over $5 \mathrm{~d}$ extinguished the conditioned fear response. During the last extinction session, memory expression was comparable to animals that received ANI after the first learning (compare LTM1 for both groups to LTM1 for groups ANI-VEH and ANI-AP5 in Fig. 2B). (C) AP5 infusions do not block the second contextual fear conditioning after the conditioned response for the first learning was extinguished (LTM2 for group VEH-AP5). The second conditioning's requirement for AP5 thus is opposite to the one following amnesia induction by ANI infusions (Fig. 2C).

context steadily declined during this time, $F_{(2,24)}=15.4, P<$ 0.0001 , in both groups, $F<1$, and by the fourth day was as low as in those animals that had received AP5 before the first contextual fear conditioning in Experiment $1, F<1.6$, or ANI after the first conditioning in Experiment 2, $F<1.8$.

One day later, animals were fear conditioned to the second context. One group received dHPC AP5 infusions before training, the other one vehicle. The retention test administered the next day revealed no significant group differences (Fig. 3C, $F_{(1,12)}=$ 2.92, $P>0.1$ ) - unlike in Experiment 2, AP5 did not block learning. This result corroborates our conclusion that the amnesia obtained in Experiment 2 by ANI infusions reflects actual memory loss, and not a retrieval inhibition of existing memory.

\section{Discussion}

Infusing ANI into the dHPC after contextual fear conditioning produced a behavioral impairment consistent with the view that disrupted consolidation, not retrieval, underpins this form of experimentally induced amnesia. Our paradigm is based on the phenomenon that NMDAr are required in the dHPC when a hippocampus-dependent task, such as contextual fear conditioning or the allocentric version of the watermaze, is learned for the first, but not the second, time (Bannerman et al. 1995; Sanders and Fanselow 2003). The storage impairment view, which attributes amnesia to memory loss, therefore predicts that infusing the NMDAr antagonist AP5 in the dHPC will block the second learning in amnesic animals, as the second learning represents the first instance of such learning for amnesic animals. The retrieval impairment view, on the other hand, predicts that the second learning in amnesic animals will not be blocked by AP5 infusions, as their amnesia represents the inability to retrieve available memory, which thus is present during the second learning.

First, we replicated that only the initial, but not subsequent, learning is affected by dHPC AP5 infusions (Experiment 1). Animals that had intact memory for the first readily learned the second conditioning despite prior AP5 infusions. In addition,

animals that had impaired memory for the first learning because of prior AP5 infusions did not acquire the second learning after receiving AP5. These results lend further support to the well-accepted view that blockade of NMDAr prevents memory acquisition, such that animals treated with AP5 before the first conditioning will have no corresponding memory during the second training, and will thus treat it as if it were the first. Second, we found that, similar to AP5, dHPC ANI infusions after the first contextual fear conditioning rendered the second learning sensitive to AP5 (Experiment 2). Consequently, amnesia for the first learning following ANI treatment is also best characterized as a storage deficit. A retrieval deficit is unlikely the reason for this impairment. Retrieval impairment views of amnesia assume that memory for the first conditioning was fully stored at the time ANI was given, such that the resulting amnesia was due to the disruption of processes associated with implementing mechanisms that allow later memory retrieval. Therefore, memory for the first conditioning should have been present during the second learning. As we have shown in Experiment 1, when memory is present, the second learning is not affected by AP5. However, this was not the case here-AP5 infusions impaired the second fear conditioning, which indicates that memory for the first learning indeed was lost because ANI disrupted its storage. Third, we found that after delayed extinction of the first contextual fear, which is thought to inhibit memory, or the conditioned response, instead of erasing it (Rescorla 2000; Bouton 2004), the second contextual fear was acquired despite AP5 infusions (Experiment 3).

In summary, AP5 (Experiment 1) and ANI (Experiment 2) had the opposite effect on the second learning's sensitivity to AP5 than extinction (Experiment 3). Although it is still unclear how ANI impairs memory, it is a broadly used amnesic agent, and its ability to induce amnesia is not debated (e.g., Canal et al. 2007; Klann and Sweatt 2008). Thus, the specific mechanism is irrelevant for the issue at hand, namely whether the resulting amnesia reflects disrupted retrieval or impaired storage: Our results suggest the latter is the case.

A possible alternative post hoc interpretation of our data could speculate that ANI indeed induces a retrieval impairment, which renders the second learning NMDAr-independent. This, however, seems unlikely for two reasons. First, since AP5 and ANI induced the same effects on the second learning, this view is forced to conclude that AP5, like ANI, caused retrieval impairment for the first learning as well. To argue, however, that memory acquisition thus was not mediated by NMDAr, as NMDAr only affected the retrieval of existing memory, would also require to fundamentally revise the accepted role of NMDAr in learning and plasticity (e.g., Nakazawa et al. 2004; Morris 2006). Second, since delayed extinction of the first contextual fear had exactly the opposite effect on the second learning as both AP5 and ANI, this account would need to assume that delayed extinction leads to a storage impairment, which is hard to reconcile with a large body of data supporting the position that delayed extinction inhibits retrieval of existing memory (e.g., Rescorla 2000; Bouton 2004). Thus, this alternative explanation of our findings appears speculative.

In this context the question arises whether attenuated freezing to the conditioned stimulus after extinction learning is due to inhibition of retrieval of the association (Bouton 1993), or inhibition of expression (Rescorla 2001) despite successful retrieval of the conditioned response, or to both, that is, partial retrieval and thus partial expression. We find that animals do not express the conditioned response fully after extinction, but they do express some freezing, unlike naïve animals that have never been contextually fear conditioned. After extinction in Experiment 3, the second learning of contextual fear did not require NMDAr, unlike the first learning. Thus, it appears that, at 
a minimum, full behavioral expression is not necessary to render the second learning NMDAr independent; additionally, one can conclude that this partial expression necessitated some form of retrieval of the original response, either partially or fully, as otherwise no freezing should have been observed. In light of the fact that the residual level of freezing after extinction (Experiment 3) is comparable to the level of freezing exhibited by animals after dHPC ANI infusion (Experiment 2), it also appears that partial expression (and thus at least partial retrieval) of the fear response alone is not sufficient to render second learning NMDArindependent-otherwise, second learning in the ANI-infused amnesic animals should have been NMDAr-independent as well, which was not the case (Experiment 2). Therefore, the quality of memory after ANI infusion appears different from the quality of memory after extinction-although both procedures induce a comparable behavioral result, they do not lead to a comparable mnemonic effect. Thus, while our study was not designed to determine the nature of extinction, our results, at the very least, strongly suggest that extinction and ANI infusion, although resulting in comparable behavior, do so by different mnemonic processes, as indicated by the different involvement of NMDAr in second learning of contextual fear following these procedures. This difference was predicted as well as detected by our paradigm.

Our results further raise the related question about what type of knowledge animals acquire that underpins the residual fear response we observe after ANI or AP5 infusions into dHPC. In these amnesic animals the second learning, like the first learning in naïve animals, required NMDAr, although these animals, unlike naive ones, expressed some contextual fear prior to second learning. Reflecting on this issue, Bannerman et al. (1995) noted that animals learn more in the watermaze task than the location of the platform. For example, the animals acquired knowledge about how to climb on the platform, visual information regarding the test environment, and so on. Likewise, in contextual fear conditioning, animals can be expected to learn more than to fear a certain environment. As Barrientos et al. (2002) point out in a report on the immediate shock deficit, animals likely acquire knowledge about the general procedure, such as being transported out of their colony, and being handled in a certain way. Dorsal hippocampus, the region we targeted with AP5 and ANI, is unlikely to mediate this knowledge. Thus, the incomplete amnesia that we (and others) observed after inducing amnesia by manipulations of the hippocampus could reflect the existence of extrahippocampal noncontextual knowledge that is able to trigger some residual fear (e.g., like our AP5-AP5 animals in Experiment 1 [Fig.1B,C], or like our ANI-AP5 animals in Experiment 2 [Fig. $2 \mathrm{~B}, \mathrm{C}])$. This knowledge, although expressed in terms of a fear response, might be different in quality from the knowledge that drives a full-fledged fear response in intact animals: While the amnesic animals experience some form of generalized noncontextual anxiety, the latter, when put back into a context, experience a specific recollection of an event in which they received a painful foot shock in a certain type of environment. This knowledge about where or even that a foot shock was experienced previously seems impaired in our amnesic animals. Thus, animals with (admittedly partial) amnesia for the first contextual fear conditioning behave like naïve animals, as they have no access to a record of experiencing a foot shock in a box.

At first sight, our findings appear at odds with the results of de $\mathrm{Hoz}$ et al. (2004), who suggest that amnesia induced by hippocampal lesions after place learning in the allocentric version of the watermaze reflects disrupted memory retrieval. In their experiments, rats were first trained to find the platform, and then they received partial hippocampal lesions (sparing about a third of the dorsal region). Two weeks later, a probe trial was administered, at the end of which the platform was made available by raising it either in the original or in a novel location. This served as a reminder for the original training, and its effects on memory recovery were assessed with a second probe trial one hour later, in which the platform was raised in the same position as in the previous probe trial. Finally, the ability to acquire new spatial knowledge was examined the next day in a different watermaze. The two central findings were (1) that rats with partial lesions were not able to remember the platform location in the first probe trial but were able to do so in the second one, irrespective of whether the platform was raised in the old or a new location, and (2) that these animals could not learn to locate a platform in a different watermaze. Animals with complete lesions of the hippocampus neither benefited from the reminder nor could be retrained.

Thus, it appears that again a situation arose in which one data set strongly supports the position that amnesia is due to impaired memory storage, while strong support is provided by a different data set for the position that amnesia reflects impaired memory retrieval. However, the data might not be contradictory. The retrieval impairment account of experimental amnesia, at the most fundamental level of analysis, needs to assume basic storage processes as well, as the information required to retrieve memories, for example, the retrieval "cues" themselves, must somehow be integrated into the networks that mediate retrieval. In other words, retrieval requires the storage of information that permits retrieval. Some theories of hippocampal function characterize it as a site required for retrieval of spatial, contextual, or episodic memory (e.g., Squire et al. 1984; Teyler and DiScenna 1986; McClelland et al. 1995; Rolls and Kesner 2006), computing an "index" to memory stored in neocortex. One possible interpretation that would reconcile the results from de Hoz et al.'s (2004) study supporting a retrieval view of amnesia, and our results favoring the storage view, thus, is that, in the hippocampus, ANI (or lesions) prevents storage of representations necessary for memory retrieval. As the recovery that de $\mathrm{Hoz}$ et al. report was dependent on residual hippocampal tissue, and as no new learning of the platform location took place since reminding animals with a platform in a new location was successful, one could speculate that the reminder provided some form of input that, together with the spared memory representations in the remaining hippocampal tissue, allowed for a partial reconstruction of the original memory necessary for retrieval of the actual location (the performance in the partially lesioned animals was inferior to the performance of control animals, so the reminder did not lead to a complete restoration of the original memory). The ability of hippocampal networks to perform pattern completion given input that only partially recapitulates the original stimulus has been taken as strongly supporting its role in memory retrieval (e.g., Rudy and O'Reilly 1999; Nakazawa et al. 2002; Kirwan et al. 2005), thus providing further support for our account of de Hoz et al.'s findings. To conclude, we propose that ANI or lesions lead to actual memory loss. But how this unavailability of information will manifest itself at the behavioral level depends on the contributions of the affected brain system to the behavior of interest-in systems involved in memory retrieval, loss of information will render available content inaccessible, while in systems involved in content representation, the content itself will no longer be available.

Our study represents a novel approach toward a better understanding of experimentally induced amnesia. The paradigm is the first to make a positive prediction for the absence of memory and provides the two accounts of amnesia with opposing predictions, such that the storage and retrieval impairment views can be tested against each other. Broad generalizations concerning the nature of amnesia are, however, not indicated in light of these findings, as it is possible that some experimental manipulations induce amnesia that reflects retrieval impairment (e.g., Riccio et al. 
2006; Sara and Hars 2006), while others indeed disrupt proper memory storage. Instead, our paradigm may be used to decide between these two possibilities.

Post hoc interpretations of the data can describe the extinction effect as memory erasure and the behavioral impairments induced by AP5 and ANI as retrieval impairments. However, these post hoc interpretations do not supply any novel predictions. Therefore, they remain philosophical positions that cannot be empirically addressed. The value of our paradigm is that it allows testing the specific predictions of existing scientific models of retrieval and consolidation. Our approach can be easily expanded to other memory systems, brain areas, amnesic agents, and tasks: As long as one behavioral or neurobiological feature is distinct between the first and second learning, it can be used to determine whether learning after amnesia induction resembles the first or second time the studied system acquires certain knowledge, and thus whether the nature of the observed amnesia reflects impaired storage or retrieval.

\section{Materials and Methods}

\section{Animal subjects}

Male Sprague-Dawley rats (Charles River, Canada) were acclimated to the animal colony for 3-5 $\mathrm{d}$ and were handled before the experiment. Food and water were provided ad libitum throughout the experiment. Animals weighed 330-360 g at the time of stereotaxic surgery. The light cycle was set to 7 am (lights on) -7 pm (lights off). Experiments were performed during the lights-on phase. All procedures were in full compliance with the standards set forth by the Canadian Council on Animal Care and were approved by McGill University's Animal Care Committee.

\section{Surgery and microinjections}

Animals were anaesthetized with a mixture of xylazine $(3.33 \mathrm{mg} /$ $\mathrm{mL})$, ketamine $(55.55 \mathrm{mg} / \mathrm{mL})$, and Domitor $(0.27 \mathrm{mg} / \mathrm{mL})$ by intraperitoneal (i.p.) injection of a volume of $1 \mathrm{~mL} / \mathrm{kg}$. Using a Kopf Stereotax, three jeweler screws were implanted into the skull, and then two steel cannulae (22 gauge) were placed, aiming bilaterally at the dorsal hippocampus (A/P $-3.6 \mathrm{~mm}, \mathrm{M} / \mathrm{L} \pm 3.1$ $\mathrm{mm}, \mathrm{D} / \mathrm{V}-2.4 \mathrm{~mm}, 10^{\circ}$ away from midline). Dental cement stabilized the cannulae. Obturators inserted in the guides prevented blocking. After surgery, an intramuscle injection of analgesic (buprenorphine, $0.324 \mathrm{mg} / \mathrm{kg}$ ) was given. An i.p. injection of Antisedan $(7.5 \mathrm{mg} / \mathrm{kg})$ suspended anesthesia.

AP5 and ANI (both from Sigma-Aldrich) were used to block NMDA receptors and protein synthesis, respectively. AP5 $(2.5 \mu \mathrm{g} /$ $\mu \mathrm{L})$ was dissolved in sterile saline, ANI $(125 \mu \mathrm{g} / \mu \mathrm{L})$ in equimolar $\mathrm{HCl}$ and sterile saline. For both, $\mathrm{pH}$ was adjusted to 7.4 with equimolar $\mathrm{NaOH}$; sterile saline served as vehicle. AP5 was infused into the dorsal hippocampus $(2 \mu \mathrm{L} /$ hemisphere $)$ via a microinjector (28 gauge) connected to a Hamilton syringe with plastic tubing. Infusion took $5 \mathrm{~min}$, and the injector remained connected for 1 extra minute to allow for diffusion away from the tip of the cannula. ANI was infused using a similar set-up, except that the infusion took 8 min (Debiec et al. 2002).

\section{Apparatus}

Two different contexts were used. To decrease generalization between them, they varied along several dimensions, i.e., shape, illumination, scent, visual and tactile features, and ambient sound. Context A was a Med-Associate box $(29 \mathrm{~cm} \times 25 \mathrm{~cm} \times$ $25 \mathrm{~cm}$ ), with aluminum sidewalls. Two lights were on the right wall and one on the left. The lights flashed alternating in a rate of 1 cycle/sec. A plastic inlet was placed into the box to create a curved back wall. A black-and-white-striped wallpaper (stripes $2.5 \mathrm{~cm}$ wide) was placed on the front wall. The floor was a slanted $\left(7^{\circ}\right)$ stainless steel grid (rod radius $1 \mathrm{~mm}$, spaced $0.5 \mathrm{~cm}$ apart) that delivered the foot shock. Wood-chip bedding barely covered the grid. Peppermint scent was sprayed onto the curved plastic wall before the animal was put in the box. A fan provided a constant background noise. A digital camera was mounted on the ceiling and videotaped the sessions for later analysis.

Context B was a Coulbourn box $(30 \mathrm{~cm} \times 26 \mathrm{~cm} \times 33 \mathrm{~cm})$ placed into a soundproof chamber. One wall was made of stainless steel and had a light bulb providing dim light. The other walls were made of colorless Plexiglas. The floor was a level stainless steel grid (bar radius $2.5 \mathrm{~mm}$, spread $1 \mathrm{~cm}$ apart). Vanilla scent was sprayed into one corner of the box before the animal was put into it. A digital cameral in front of the box recorded the sessions.

\section{Behavioral procedures}

\section{Pilot study}

The pilot study aimed to establish effectivity of the conditioning parameters and lack of generalization between contexts. Replicating the basic protocol of Sanders and Fanselow (2003), animals $(N=8)$ received the first contextual fear conditioning in Context A or B. They were placed in the box for $4 \mathrm{~min} 30 \mathrm{sec}$. The first foot shock (Context A: scrambled $1.2 \mathrm{~mA}, 1 \mathrm{sec}$; Context B: unscrambled 1 $\mathrm{mA}, 1 \mathrm{sec}$ ) was delivered after $3 \mathrm{~min}$, the second one $30 \mathrm{sec}$ later. Twenty-four hours later, animals were tested without shocks in the same context for $4 \mathrm{~min}$. Four days later, animals received the second training (those that were first conditioned in A were now conditioned in $\mathrm{B}$, and vice versa). Foot shocks were delivered as before, and memory was assessed $24 \mathrm{~h}$ later. The results were threefold: (1) Animals acquired contextual fear to the first context, freezing significantly more during test $(79 \%$, SEM $=7.8)$ than during training before the first shock was delivered $(0 \%), F_{(1,7)}=$ 85.2, $P<0.0001 ;(2)$ only very little fear generalization was observed to the second context, as freezing prior to shock delivery $(15 \%$, SEM $=9.5)$ was not significantly different from preshock freezing to the first context, $F_{(1,7)}=2.6, P=0.15$; and (3) a training effect was absent, as freezing during the first and second test was comparable, although it was slightly higher during the latter, indicating a possible small savings effect, $F_{(1,7)}=4.2, P=0.08$.

\section{Experiment 1: Acquisition blockade}

One week after surgery, animals were fear conditioned as described above (i.e., half were trained and tested first in $\mathrm{A}$, then in $\mathrm{B}$, and vice versa). Immediately before training, animals were infused with AP5 or vehicle. Drug treatment was randomly assigned. Four
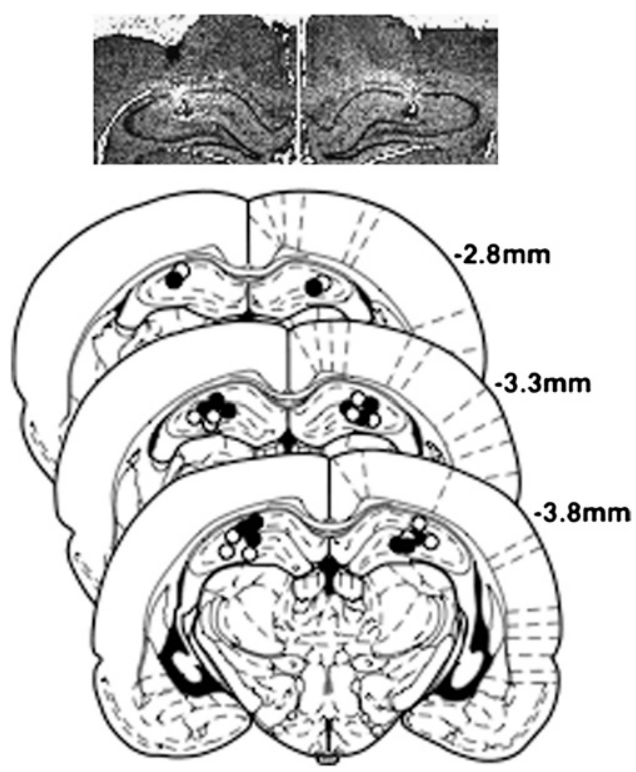

Figure 4. Histology for Experiment 3. Photograph shows typical placement of cannulae in dHPC. Bottom three images show placements for all animals. 
days later, immediately before the second training, half of the initially vehicle-treated animals received either vehicle or AP5 (VEH-VEH, $N=5$; VEH-AP5, $N=6$ ), as did the initially AP5-treated animals (Veh-AP5, $N=6$; AP5-AP5, $N=5$ ).

\section{Experiment 2: Consolidation blockade}

The protocol was similar to Experiment 1, but in the first conditioning animals received infusions (either ANI or vehicle) after, not before training. Four groups were used (VEH-VEH, $N=5$; VEH-AP5, $N=5$; ANI-VEH, $N=7$; and ANI-AP5, $N=5$ ). To test whether animals in the ANI-AP5 group did not suffer hippocampal damage as a consequence of the infusions animals were retrained in a new context, $2 \mathrm{~d}$ after the final test, but without infusions.

\section{Experiment 3: Extinction}

The protocol was similar to Experiment 1, however, all animals received vehicle infusions before the first training. In the $5 \mathrm{~d}$ following training, animals received a daily 4-min extinction test in the first learning context. One day after the last session, animals received vehicle $(N=7)$ or AP5 $(N=7)$ infusion before the second conditioning. Memory for the second conditioning was tested $24 \mathrm{~h}$ later.

\section{Behavior measurement and statistics}

Freezing (immobilization except for respiratory movements) was measured as the conditioned response by a trained scorer blind to the experimental condition. Freezing was measured during the 4min memory test in blocks of $30 \mathrm{sec}$. As no linear or quadratic trend was found in the data, scores from the eight blocks were averaged. Results were analyzed with ANOVA and Tukey's post hoc tests, with Type-I error of $\alpha=0.05$.

\section{Histology}

Animals were deeply anaesthesized, then received dHPC infusion of Eosin red dye (volume and rate identical to AP5), and were decapitated. Brains were fixed in a mixture of $4 \%$ paraformaldehyde and 30\% sucrose-saline. A cryostat obtained sections of 50$\mu \mathrm{m}$ thickness. Cannula placement was checked under a light microscope. Animals were included in the analysis when both cannulae were in the dHPC (Fig. 4).

\section{Acknowledgments}

This work was supported by grants awarded to K.N. from the Natural Sciences and Engineering Research Council of Canada (NSERC), Canadian Institutes of Health Research (CIHR), the Volkswagen Foundation, and the EJLB Foundation.

\section{References}

Bailey, C.H., Bartsch, D., and Kandel, E.R. 1996. Toward a molecular definition of long-term memory storage. Proc. Natl. Acad. Sci. 93: 13445-13452.

Bannerman, D.M., Good, M.A., Butcher, S.P., Ramsay, M., and Morris, R.G. 1995. Distinct components of spatial learning revealed by prior training and NMDA receptor blockade. Nature 378: 182-186.

Barrientos, R.M., O'Reilly, R.C., and Rudy, J.W. 2002. Memory for context is impaired by injecting anisomycin into dorsal hippocampus following context exploration. Behav. Brain Res. 134: 299-306.

Bouton, M.E. 1993. Context, time, and memory retrieval in the interference paradigms of Pavlovian learning. Psychol. Bull. 114: 80-99.

Bouton, M.E. 2004. Context and behavioral processes in extinction. Learn. Mem. 11: 485-494.
Canal, C.E., Chang, Q., and Gold, P.E. 2007. Amnesia produced by altered release of neurotransmitters after intraamygdala injections of a protein synthesis inhibitor. Proc. Natl. Acad. Sci. 104: 12500-12505.

Davis, H.P. and Squire, L.R. 1984. Protein synthesis and memory: A review. Psychol. Bull. 96: 518-559.

de Hoz, L., Martin, S.J., and Morris, R.G. 2004. Forgetting, reminding, remembering: The retrieval of lost spatial memory. PLoS Biol. 2: E225. doi: 10.1371/journal.pbio.0020225.

Debiec, J., LeDoux, J.E., and Nader, K. 2002. Cellular and systems reconsolidation in the hippocampus. Neuron 36: 527-538.

Gold, P.E. and King, R.A. 1974. Retrograde amnesia: Storage failure versus retrieval failure. Psychol. Rev. 81: 465-469.

Gordon, W.C. and Mowrer, R.R. 1980. An extinction trial as a reminder treatment following electroconvulsive shock. Anim. Learn. Behav. 8: 363-367.

Kirwan, C.B., Gilbert, P.E., and Kesner, R.P. 2005. The role of the hippocampus in the retrieval of a spatial location. Neurobiol. Learn. Mem. 83: 65-71.

Klann, E. and Sweatt, D. 2008. Altered protein synthesis is a trigger for longterm memory formation. Neurobiol. Learn. Mem. 89: 247-259.

Lewis, D.J. 1979. Psychobiology of active and inactive memory. Psychol. Bull. 86: 1054-1083.

McClelland, J.L., McNaughton, B.L., and O'Reilly, R.C. 1995. Why there are complementary learning systems in the hippocampus and neocortex: Insights from the successes and failures of connectionist models of learning and memory. Psychol. Rev. 102: 419-457.

Miller, R.R. and Marlin, N.A. 1984. The physiology and semantics of consolidation: Of mice and men. In Memory consolidation: Psychobiology of cognition (eds. H. Weingartner and E.S. Parker), pp. 85-109. Erlbaum, Hillsdale, NJ.

Morris, R.G.M. 2006. Elements of a neurobiological theory of hippocampal function: The role of synaptic plasticity, synaptic tagging and schemas. Eur. J. Neurosci. 23: 2829-2846.

Nader, K. and Wang, S.H. 2006. Fading in. Learn. Mem. 13: 530-535.

Nakazawa, K., Quirk, M.C., Chitwood, R.A., Watanabe, M., Yeckel, M.F., Sun, L.D., Kato, A., Carr, C.A., Johnston, D., Wilson, M.A., et al. 2002. Requirement for hippocampal CA3 NMDA receptors in associative memory recall. Science 297: 211-218.

Nakazawa, K., McHugh, T.J., Wilson, A.W., and Tonegawa, S. 2004. NMDA receptors, place cells and hippocampal spatial memory. Nat. Rev. Neurosci. 5: 361-372.

Philips, G.T., Tzvetkova, E.I., Marinesco, S., and Carew, T.J. 2006. Latent memory for sensitization in Aplysia. Learn. Mem. 13: 224-229.

Rescorla, R.A. 2000. Extinction can be enhanced by a concurrent excitor. $J$. Exp. Psychol. Anim. Behav. Process. 26: 251-256.

Rescorla, R.A. 2001. Experimental extinction. In Handbook of contemporary learning theories (eds. R.R. Mowrer and S.B. Klein), pp. 119-154. Erlbaum, Hillsdale, NJ.

Riccio, D.C., Millin, P.M., and Bogart, A.R. 2006. Reconsolidation: A brief history, a retrieval view, and some recent issues. Learn. Mem. 13: 536544.

Rolls, E.T. and Kesner, R.P. 2006. A computational theory of hippocampal function, and empirical tests of the theory. Prog. Neurobiol. 79: 1-48.

Rudy, J.W. and O’Reilly, R.C. 1999. Contextual fear conditioning, conjunctive representations, pattern completion, and the hippocampus. Behav. Neurosci. 113: 867-880.

Sanders, M.J. and Fanselow, M.S. 2003. Pre-training prevents context fear conditioning deficits produced by hippocampal NMDA receptor blockade. Neurobiol. Learn. Mem. 80: 123-129.

Sara, S.J. 1973. Recovery from hypoxia and ECS-induced amnesia after a single exposure to training environment. Physiol. Behav. 10: 85-89.

Sara, S.J. and Hars, B. 2006. In memory of reconsolidation. Learn. Mem. 13: $515-521$.

Squire, L.R. 2006. Lost forever or temporarily misplaced? The long debate about the nature of memory impairment. Learn. Mem. 13: 522-529.

Squire, L.R., Cohen, J., and Nadel, L. 1984. The medial temporal region and memory consolidation: A new hypothesis. In Memory consolidation: Psychobiology of cognition (eds. H. Weingartner and E.S. Parker), pp. 185210. Erlbaum, Hillsdale, NJ.

Teyler, T.J. and DiScenna, P. 1986. The hippocampal memory indexing theory. Behav. Neurosci. 100: 147-152.

Received October 23, 2008; accepted in revised form January 31, 2009. 


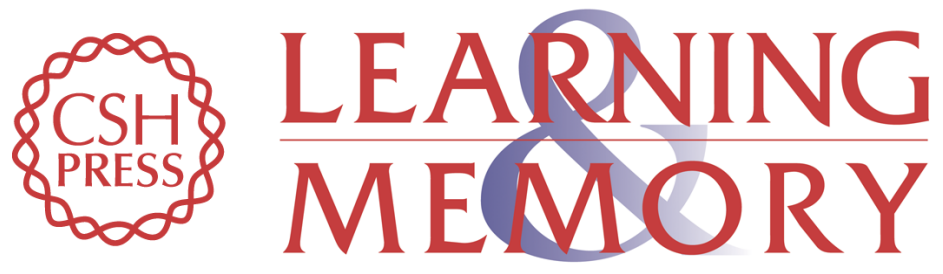

\section{Storage or retrieval deficit: The yin and yang of amnesia}

Oliver Hardt, Szu-Han Wang and Karim Nader

Learn. Mem. 2009, 16:

Access the most recent version at doi:10.1101//m.1267409

References This article cites 29 articles, 9 of which can be accessed free at: http://learnmem.cshlp.org/content/16/4/224.full.html\#ref-list-1

License

Email Alerting Receive free email alerts when new articles cite this article - sign up in the box at the Service top right corner of the article or click here. 